\title{
SOME REMARKS ON TRANSPORTATION COST AND RELATED INEQUALITIES
}

\author{
MARK W. MECKES
}

\begin{abstract}
We discuss transportation cost inequalities for uniform measures on convex bodies, and connections with other geometric and functional inequalities. In particular, we show how transportation inequalities can be applied to the slicing problem, and prove a new log-Sobolev-type inequality for bounded domains in $\mathbb{R}^{n}$.
\end{abstract}

\section{INTRODUCTION}

We work in $\mathbb{R}^{n}$ equipped with its standard inner product $\langle\cdot, \cdot\rangle$ and Euclidean norm $|\cdot|$. $|A|$ also denotes the volume (Lebesgue measure) of a measurable set $A . D_{n}$ is the Euclidean ball of volume one. For a measurable set $A$ with $0<|A|<\infty, m_{A}$ denotes the uniform probability measure on $A$, that is, $m_{A}(B)=\frac{|A \cap B|}{|A|}$. The symbols $\mu$ and $\nu$ will always stand for Borel probability measures on $\mathbb{R}^{n}$.

We first introduce two different ways to quantify the difference between two probability measures. First, for $p \geq 1$, the $\left(L_{p}\right)$ Wasserstein distance between $\mu$ and $\nu$ is

$$
W_{p}(\mu, \nu)=\inf _{\pi}\left(\int|x-y|^{p} d \pi(x, y)\right)^{1 / p},
$$

where $\pi$ runs over probability measures on $\mathbb{R}^{n} \times \mathbb{R}^{n}$ with marginals $\mu$ and $\nu$. We will be interested mainly in the special cases $p=1,2$. Second, if $\nu \ll \mu$, the relative entropy of $\nu$ with respect to $\mu$ is

$$
H(\nu \mid \mu)=\int \log \left(\frac{d \nu}{d \mu}\right) d \nu
$$

The $\left(L_{p}\right)$ transportation cost constant $\tau_{p}(\mu)$ is the largest constant $\tau$ such that

$$
W_{p}(\mu, \nu) \leq \sqrt{\frac{2}{\tau} H(\nu \mid \mu)}
$$

for every $\nu \ll \mu$. An inequality of the form of (II) is referred to as a transportation cost inequality for $\mu$. Note that if $p \leq q$, then $W_{p} \leq W_{q}$ by Hölder's inequality, and hence $\tau_{p}(\mu) \geq \tau_{q}(\mu)$.

Transportation cost inequalities are by now well known as a method to derive measure concentration (cf. [8, Chapter 6]). In fact, as follows from Bobkov and Götze's dual characterization of the $L_{1}$ transportation cost inequality [3], $\tau_{1}(\mu)$ is equivalent to the best constant $\alpha$ in the normal concentration inequality:

$$
\mu\left(\left\{x \in \mathbb{R}^{n}:|F(x)| \geq t\right\}\right) \leq 2 e^{-\alpha t^{2}} \text { for } t>0
$$

for all 1-Lipschitz functions $F$ with $\int F d \mu=0$. 
In this paper we consider transportation cost inequalities for uniform measures on convex bodies. In the next section we show that such inequalities can be applied to the slicing problem. In the last section we discuss their relationship with Sobolev-type functional inequalities, and present a logarithmic Sobolev inequality with trace for bounded domains in $\mathbb{R}^{n}$.

\section{Relation to THE SLICING PROBlem}

We recall the following definitions and facts about isotropic convex bodies (see [10]). A convex body $K$ is called isotropic if

(1) its centroid is 0 ,

(2) $|K|=1$, and

(3) there is a constant $L_{K}>0$ such that

$$
\int_{K}\langle x, y\rangle^{2} d x=L_{K}^{2}|y|^{2}
$$

for all $y \in \mathbb{R}^{n}$.

Every convex body $K$ has an affine image $T(K)$ (unique up to orthogonal transformations) which is isotropic; the isotropic constant of $K$ is defined as $L_{K}=L_{T(K)}$. The isotropic constant also has the extremal characterization

$$
L_{K}=\min _{T}\left(\frac{1}{n|K|^{1+2 / n}} \int_{T(K)}|x|^{2} d x\right)^{1 / 2},
$$

where $T$ runs over volume-preserving affine transformations of $\mathbb{R}^{n}$, with equality iff $K$ is isotropic. The slicing problem for convex bodies asks whether there is a universal constant $c$ such that $L_{K} \leq c$ for all convex bodies $K$; see [10] for extensive discussion and alternate formulations.

If $K, B \subset \mathbb{R}^{n}$ are convex bodies, the volume ratio of $K$ in $B$ is

$$
\operatorname{vr}(B, K)=\min _{T}\left(\frac{|B|}{|T(K)|}\right)^{1 / n},
$$

where $T$ runs over affine transformations of $\mathbb{R}^{n}$ such that $T(K) \subset B$. The following lemma indicates the relevance of transportation cost inequalities to the slicing problem.

Lemma 1. Let $K, B \subset \mathbb{R}^{n}$ be convex bodies, with $B$ isotropic. Then

$$
L_{K} \leq c(1+\sqrt{\log v}) v \tau^{-1 / 2}
$$

where $\tau=\tau_{1}(B), v=\operatorname{vr}(B, K)$, and $c$ is an absolute constant.

Proof. We may assume that $K \subset B$ and $|K|=v^{-n}$. If $\delta_{0}$ denotes the point mass at $0 \in \mathbb{R}^{n}$, then by the triangle inequality for $W_{1}$,

$$
\begin{aligned}
\frac{1}{|K|} \int_{K}|x| d x & =W_{1}\left(m_{K}, \delta_{0}\right) \leq W_{1}\left(m_{K}, m_{B}\right)+W_{1}\left(m_{B}, \delta_{0}\right) \\
& \leq \sqrt{\frac{2}{\tau} H\left(m_{K} \mid m_{B}\right)}+\int_{B}|x| d x
\end{aligned}
$$




$$
\begin{aligned}
& \leq \sqrt{\frac{2}{\tau} \log \frac{1}{|K|}}+\left(\int_{B}|x|^{2} d x\right)^{1 / 2} \\
& =\sqrt{\frac{2 n}{\tau} \log v}+\sqrt{n} L_{B} .
\end{aligned}
$$

Now by applying (2) to a linear functional, $L_{B} \leq c \tau^{-1 / 2}$. On the other hand, by Borell's lemma (see e.g. [8, Section 2.2]), there is an absolute constant $c$ such that

$$
\left(\frac{1}{|K|} \int_{K}|x|^{2} d x\right)^{1 / 2} \leq c \frac{1}{|K|} \int_{K}|x| d x .
$$

The claim now follows from the extremal characterization of $L_{K}$ (3).

An analogous estimate with $\tau=\tau_{2}(B)$ can be proved more directly, without Borell's lemma.

In light of the equivalence of $L_{1}$ transportation cost inequalities and normal concentration, Lemma 1 can also be thought of as an application of measure concentration to the slicing problem. Since the Euclidean ball is well known to have normal concentration, as an immediate corollary we obtain the following known fact.

Corollary 2. If $\operatorname{vr}\left(D_{n}, K\right) \leq c$, then $L_{K} \leq c^{\prime}$, where $c^{\prime}$ depends only on $c$.

Recently, Klartag [7] introduced the following isomorphic version of the slicing problem: given a convex body $K$, is there a convex body $B$ such that $L_{B} \leq c_{1}$ and $d(B, K) \leq c_{2}$, where $d$ is Banach-Mazur distance? In the case that $K$ and $B$ are centrally symmetric, Klartag solved this problem in the affirmative, up to a logarithmic (in $n$ ) factor in $c_{2}$. Lemma 1 suggests approaching the slicing problem via a modified version of the isomorphic problem: given a convex body $K$, can one find a "similar" body $B$ such that $\tau_{1}(B)$ is large when $B$ is in isotropic position? Notice that while Klartag's result uses Banach-Mazur distance to quantify "similarity" of bodies, in Lemma 1 it is the weaker measure of volume ratio which is relevant. It also seems that this approach via transportation cost is less sensitive to central symmetry than more traditional methods of asymptotic convexity.

Finally, we remark that the real point of the proof of Lemma 1 is that moments of the Euclidean norm on convex bodies, thought of as functionals of the bodies, are Lipschitz with respect to Wasserstein distances on uniform measures. This suggests an alternative approach to the slicing problem, related to the one discussed above, of directly studying optimal (or near-optimal) probability measures $\pi$ in the definition of $W_{p}\left(m_{K}, m_{B}\right)$ for $p=1,2$. Particularly in the case $p=2$ a great deal is known about the optimal $\pi$; see [12 for an excellent survey.

\section{Functional inequalities}

The entropy of $f: \mathbb{R}^{n} \rightarrow \mathbb{R}_{+}$with respect to $\mu$ is

$$
\operatorname{Ent}_{\mu}(f)=\int f \log \left(\frac{f}{\int f d \mu}\right) d \mu,
$$


and the variance of $f: \mathbb{R}^{n} \rightarrow \mathbb{R}$ with respect to $\mu$ is

$$
\operatorname{Var}_{\mu}(f)=\int f^{2} d \mu-\left(\int f d \mu\right)^{2} .
$$

The logarithmic Sobolev constant $\rho(\mu)$ is the largest constant $\rho$ such that

$$
\operatorname{Ent}_{\mu}\left(f^{2}\right) \leq \frac{2}{\rho} \int|\nabla f|^{2} d \mu
$$

for all smooth $f \in L_{2}(\mu)$. The spectral gap $\lambda(\mu)$ is the largest constant $\lambda$ such that

$$
\operatorname{Var}_{\mu}(f) \leq \frac{1}{\lambda} \int|\nabla f|^{2} d \mu
$$

for all smooth $f \in L_{2}(\mu)$. It is well known (cf. [8] ) that a logarithmic Sobolev inequality for $\mu$ implies normal concentration (and hence an $L_{1}$ transportation cost inequality, by Bobkov and Götze's result [3]) and a spectral gap inequality implies exponential concentration. A result of Otto and Villani [1] shows further that

$$
\rho(\mu) \leq \tau_{2}(\mu) \leq \lambda(\mu)
$$

for any absolutely continuous $\mu$. Thus transportation cost inequalities are somehow intermediate between these Sobolev-type functional inequalities, and it is of interest here to consider what is known about $\rho(K)$ and $\lambda(K)$ for a convex body $K$. We briefly review known results.

Kannan, Lovász, and Simonovits [6] showed that

$$
\lambda(K) \geq c\left(\frac{1}{|K|} \int_{K}|x-z|^{2} d x\right)^{-1},
$$

where $z$ is the centroid of $K$. It is easy to see that this is an optimal estimate in general. By testing (5) on linear functionals, one can see that $\lambda(\mu) \leq \alpha_{1}^{-1}$ for any $\mu$, where $\alpha_{1}$ is the largest eigenvalue of the covariance matrix of $\mu$. If $\alpha_{1}$ is much larger than the remaining eigenvalues (i.e., $\mu$ is close to being one-dimensional), then $\int|x|^{2} d \mu(x) \approx \alpha_{1}$. However, this situation is far from isotropicity (in which all the eigenvalues are equal), and the authors of [6] conjecture that when $K$ is isotropic,

$$
\lambda(K) \geq c n\left(\int_{K}|x|^{2} d x\right)^{-1}=\frac{c}{L_{K}^{2}} .
$$

Bobkov [2] estimated $\rho(K)$ in terms of the $L_{\psi_{2}}\left(m_{K}\right)$ norm of $|\cdot|$; in the case that $K$ is isotropic, this can be combined with a result of Alesker [1] to yield

$$
\rho(K) \geq \frac{c}{n L_{K}^{2}} .
$$

The estimate for $\tau_{1}(K)$ which follows from (7) also follows by combining Alesker's result with an $L_{1}$ transportation cost inequality proved recently by Bolley and Villani [5] in an extremely general setting. The estimate (7) misses the level of (6) by a factor of $n$, but in this case the estimate cannot be sharpened even when $K$ is isotropic: if $K$ is taken to be the $\ell_{1}^{n}$ unit ball, renormalized to have volume one, then exponential concentration correctly 
describes the behavior of a linear functional in a coordinate direction; it can in fact be shown that $\tau_{1}(K) \approx \frac{1}{n}$ in this case. However, in two concrete cases we have best possible estimates:

$$
\rho\left(Q_{n}\right) \geq c \text { and } \rho\left(D_{n}\right) \geq c,
$$

where $Q_{n}$ is a cube of volume 1 . The estimate for $Q_{n}$ is probably folklore; the estimate for $D_{n}$ is due to Bobkov and Ledoux [4].

Finally, we present the following "doubly homogeneous $L_{p}$ trace logarithmic Sobolev inequality" for uniform measures on bounded domains, inspired both by the search for good estimates on $\rho(K)$ for isotropic $K$, and by the recent work 9] by Maggi and Villani on trace Sobolev inequalities. This seems not to be directly comparable to the classical logarithmic Sobolev inequality (44), but interestingly is completely insensitive to isotropicity or even convexity of the domain. For $p>1$ we denote by $q$ the conjugate exponent $q=\frac{p}{p-1}$, and $\omega_{n}=\frac{\pi^{n / 2}}{\Gamma(1+n / 2)}$ is the volume of the Euclidean unit ball.

Proposition 3. Let $\Omega \subset \mathbb{R}^{n}$ be open and bounded with locally Lipschitz boundary and let $p \geq 1$. Then

$$
\operatorname{Ent}_{\Omega}\left(|f|^{p}\right) \leq\left(\frac{p-1}{n+q}\right)^{p-1} \frac{1}{\omega_{n}^{p / n}|\Omega|^{1-p / n}} \int_{\Omega}|\nabla f|^{p}+\frac{1}{\omega_{n}^{1 / n}|\Omega|^{1-1 / n}} \int_{\partial \Omega}|f|^{p}
$$

for every locally Lipschitz $f: \bar{\Omega} \rightarrow \mathbb{R}$, where $\left(\frac{p-1}{n+q}\right)^{p-1}$ is interpreted as 1 if $p=1$, and the integral over $\partial \Omega$ is with respect to $(n-1)$-dimensional Hausdorff measure.

In the case $p=2$ and $\left.f\right|_{\partial \Omega}=0$, we obtain

$$
\operatorname{Ent}_{\Omega}\left(f^{2}\right) \leq \frac{1}{(n+2) \omega_{n}^{2 / n}|\Omega|^{1-2 / n}} \int_{\Omega}|\nabla f|^{2} \leq \frac{c}{|\Omega|^{1-2 / n}} \int_{\Omega}|\nabla f|^{2} .
$$

Notice that

$$
\frac{|\Omega|^{2 / n}}{(n+2) \omega_{n}^{2 / n}}=\frac{|\Omega|^{2 / n}}{n \omega_{n}^{1+2 / n}} \int_{D_{n}}|x|^{2} d x \leq \frac{1}{n|\Omega|} \int_{\Omega}|x|^{2} d x
$$

with equality only if $\Omega$ is a Euclidean ball. Therefore if one restricts the logarithmic Sobolev inequality (4) for $\mu=m_{\Omega}$ to functions which vanish on the boundary of $\Omega$, one can improve the constant $\rho$ to $2(n+2) \omega_{n}^{2 / n}|\Omega|^{-2 / n}$, which is always stronger by at least a factor of 2 than the best possible result for general $f$, and much stronger still in many cases.

Proof of Proposition 3. The proof is based on the results of Brenier and McCann on mass transportation via a convex gradient; we refer to [12] for details and references. To begin, we assume that $p>1$; the case $p=1$ follows the same lines and is slightly simpler. We also assume that $f$ is smooth and nonnegative,

$$
\frac{1}{|\Omega|} \int_{\Omega} f^{p}=1
$$

and

$$
|\Omega|=\left(\frac{n+q}{p-1}\right)^{n / q} \omega_{n}
$$


We will use the fact that there is a convex function $\varphi$ such that $\nabla \varphi$ (the Brenier map) transports the probability measure $f^{p} d m_{\Omega}$ to the probability measure $m_{B_{R}}$, where $B_{R}=$ $|\Omega|^{1 / n} D_{n}$ is the Euclidean ball normalized so that $\left|B_{R}\right|=|\Omega|$.

By the results of McCann, the Monge-Ampère equation

$$
f^{p}(x)=\operatorname{det} H_{A} \varphi(x)
$$

is satisfied $f^{p} d m_{\Omega^{-}}$-a.e., where $H_{A} \varphi$ is the Aleksandrov Hessian of $\varphi$ (i.e., the absolutely continuous part of the distributional Hessian $H \varphi$ ). Using the fact that $\varphi$ is convex and $\log t \leq t-1$ for $t>0$,

$$
\log f^{p}(x)=\log \operatorname{det} H_{A} \varphi(x) \leq \Delta_{A} \varphi(x)-n,
$$

where $\Delta_{A} \varphi$ is the Aleksandrov Laplacian of $\varphi$ (i.e., the trace of $H_{A} \varphi$ ). Integrating with respect to $f^{p} d m_{\Omega}$ yields

$$
\frac{1}{|\Omega|} \int_{\Omega} f^{p} \log f^{p} \leq \frac{1}{|\Omega|} \int_{\Omega} f^{p} \Delta_{A} \varphi-n \leq \frac{1}{|\Omega|} \int_{\Omega} f^{p} \Delta \varphi-n,
$$

since $\Delta_{A} \varphi \leq \Delta \varphi$ as distributions, where $\Delta \varphi$ is the distributional Hessian of $\varphi$. Integrating by parts (cf. 9] for a detailed justification),

$$
\begin{aligned}
\frac{1}{|\Omega|} \int_{\Omega} f^{p} \Delta \varphi & =-\frac{1}{|\Omega|} \int_{\Omega}\left\langle\nabla \varphi, \nabla\left(f^{p}\right)\right\rangle+\frac{1}{|\Omega|} \int_{\partial \Omega}\langle\nabla \varphi, \sigma\rangle f^{p} \\
& =-\frac{p}{|\Omega|} \int_{\Omega} f^{p-1}\langle\nabla \varphi, \nabla f\rangle+\frac{1}{|\Omega|} \int_{\partial \Omega}\langle\nabla \varphi, \sigma\rangle f^{p},
\end{aligned}
$$

where $\sigma$ is the outer unit normal vector to $\partial \Omega$.

Now

$$
\frac{1}{|\Omega|} \int_{\partial \Omega}\langle\nabla \varphi, \sigma\rangle f^{p} \leq \frac{R}{|\Omega|} \int_{\partial \Omega} f^{p}=\frac{1}{\omega_{n}^{1 / n}|\Omega|^{1-1 / n}} \int_{\partial \Omega} f^{p} .
$$

On the other hand, by Hölder's inequality, the definition of mass transport, and the arithmeticgeometric means inequality,

$$
\begin{aligned}
-\frac{p}{|\Omega|} \int_{\Omega} f^{p-1}\langle\nabla \varphi, \nabla f\rangle & \leq p\left(\frac{1}{|\Omega|} \int_{\Omega} f^{p}|\nabla \varphi|^{q}\right)^{1 / q}\left(\frac{1}{|\Omega|} \int_{\Omega}|\nabla f|^{p}\right)^{1 / p} \\
& \leq \frac{p-1}{\left|B_{R}\right|} \int_{B_{R}}|x|^{q} d x+\frac{1}{|\Omega|} \int_{\Omega}|\nabla f|^{p} \\
& =\frac{(p-1) n}{n+q} R^{q}+\frac{1}{|\Omega|} \int_{\Omega}|\nabla f|^{p} \\
& =n+\left(\frac{p-1}{n+q}\right)^{p-1} \frac{1}{\omega_{n}^{p / n}|\Omega|^{1-p / n}} \int_{\Omega}|\nabla f|^{p}
\end{aligned}
$$

Combining (18), (91), (10), and (11) yields

$$
\operatorname{Ent}_{\Omega}\left(f^{p}\right) \leq\left(\frac{p-1}{n+q}\right)^{p-1} \frac{1}{\omega_{n}^{p / n}|\Omega|^{1-p / n}} \int_{\Omega}|\nabla f|^{p}+\frac{1}{\omega_{n}^{1 / n}|\Omega|^{1-1 / n}} \int_{\partial \Omega} f^{p} .
$$


Both sides of this inequality have the same homogeneity with respect to both $f$ and $\Omega$, so the claim follows for general $f$ and $\Omega$ by rescaling, approximation, and the fact that $|\nabla| f||=|\nabla f|$ a.e.

\section{ACKNOWLEDGEMENT}

The author thanks S. Bobkov for his comments on an earlier version of this paper.

\section{REFERENCES}

[1] S. Alesker. $\psi_{2}$-estimate for the Euclidean norm on a convex body in isotropic position. In Geometric Aspects of Functional Analysis (1992-1994), volume 77 of Oper. Theory Adv. Appl., pages 1-4. Birkhäuser, Basel, 1995.

[2] S.G. Bobkov. Isoperimetric and analytic inequalities for log-concave probability measures. Ann. Probab., 27(4):1903-1921, 1999.

[3] S.G. Bobkov and F. Götze. Exponential integrability and transportation cost related to logarithmic Sobolev inequalities. J. Funct. Anal., 163(1):1-28, 1999.

[4] S.G. Bobkov and M. Ledoux. From Brunn-Minkowski to Brascamp-Lieb and to logarithmic Sobolev inequalities. Geom. Funct. Anal., 10(5):1028-1052, 2000.

[5] F. Bolley and C. Villani. Weighted Csiszár-Kullback-Pinsker inequalities and applications to transportation inequalities. Ann. Fac. Sci. Toulouse. To appear.

[6] R. Kannan, L. Lovász, and M. Simonovits. Isoperimetric problems for convex bodies and a localization lemma. Discrete Comput. Geom., 13(3-4):541-559, 1995.

[7] B. Klartag. An isomorphic version of the slicing problem. J. Funct. Anal. To appear.

[8] M. Ledoux. The Concentration of Measure Phenomenon, volume 89 of Mathematical Surveys and Monographs. American Mathematical Society, Providence, RI, 2001.

[9] F. Maggi and C. Villani. Balls have the worst best Sobolev inequalities. Preprint, 2004.

[10] V.D. Milman and A. Pajor. Isotropic position and inertia ellipsoids and zonoids of the unit ball of a normed $n$-dimensional space. In Geometric Aspects of Functional Analysis (1987-88), volume 1376 of Lecture Notes in Math., pages 64-104. Springer, Berlin, 1989.

[11] F. Otto and C. Villani. Generalization of an inequality by Talagrand and links with the logarithmic Sobolev inequality. J. Funct. Anal., 173(2):361-400, 2000.

[12] C. Villani. Topics in Optimal Transportation, volume 58 of Graduate Studies in Mathematics. American Mathematical Society, Providence, RI, 2003.

E-mail address: mark@math.stanford.edu

Department of Mathematics, Stanford University, Stanford, California 94305, U.S.A. 